\title{
SÍNTESE DE NANOPARTÍCULAS DE PRATA UTILIZANDO POLÍMEROS IÔNICOS E NÃO IÔNICOS
}

\author{
C. H. RODRIGUES ${ }^{1}$,A. FONTES ${ }^{2}$, J.S.A. FALCÃO ${ }^{3}$, G.PEREIRA ${ }^{4}$ e B. S. SANTOS ${ }^{1}$ \\ ${ }^{1}$ Departamento de Ciências Farmacêuticas, Universidade Federal de Pernambuco \\ ${ }^{2}$ Departamento de Biofísica e Radiobiologia, Universidade Federal de Pernambuco \\ ${ }^{3}$ Curso de Farmácia, Centro de Educação e Saúde, Universidade Federal de Campina Grande \\ ${ }^{4}$ Departamento de Química Fundamental, Universidade Federal de Pernambuco \\ claudiohrodrigues@live.com
}

\begin{abstract}
RESUMO - Entre os sistemas coloidais mais estudados estão as nanopartículas de prata (AgNPS), que podem ser sintetizadas por diversas metodologias. $O$ método de crescimento conhecido como o "crescimento por semente" é rápido, reprodutivel e as partículas são obtidas pela redução controlada dos ions $\mathrm{Ag}^{+}$. Através da adaptação desta metodologia conhecida, alterou-se os polímeros na sintese das sementes de prata para posterior crescimento, observando o modelo pelo qual esse crescimento ocorre, além da comparação realizada com o método original, que origina partículas de $20-38 \mathrm{~nm}$. Observou-se a direta influência do polímero e de sua carga na semente para o modelo de crescimento estabelecido, assim como a eficácia do método em produzir partículas hábeis para diversas aplicações.
\end{abstract}

Palavras chave: Sistemas coloidais, Nanomateriais, Espectroscopia

\begin{abstract}
Among the most studied colloidal systems are silver nanoparticles (AgNPs), which can be synthesized by several methodologies. The growth method known as "seed growth" is fast, reproducible and the particles are obtained by the controlled reduction of $\mathrm{Ag}^{+}$ions. By adapting this known methodology, the polymers were altered in the silver seed synthesis for further growth, observing the model by which this growth occurred, in addition to the comparison with the original method, that gives particles of 20-38 $\mathrm{nm}$. The direct influence of the polymer and its load on the seed was observed for the established growth model, as well as the effectiveness of the method in producing seed particles for various applications.
\end{abstract}

Keywords: Colloidal systems, Nanomaterials, Spectroscopy

\section{INTRODUÇÃO}

Um sistema coloidal apresenta uma fase dispersa $(1 \mathrm{~nm}$ a $1 \mu \mathrm{m})$ e outra dispersante. Um dos sistemas coloidais bastante estudados atualmente são nanopartículas metálicas crescidas em meio líquido, em virtude de suas propriedades ópticas, plasmônicas, biomédicas 
e catalíticas. As nanopartículas de Prata (AgNPs) são sistemas coloidais $(5-100 \mathrm{~nm})$ formados através dos fenômenos de nucleação e crescimento. A nucleação é a etapa onde são formados os pequenos aglomerados (núcleos ou sementes de prata) que surgem pela redução de íons $\mathrm{Ag}^{+}$por agentes redutores (por ex. borohidreto de sódio), resultando em agregados (Ag-Ag)n. A segunda etapa de formação das AgNPs ocorre pelo crescimento destes agregados para partículas maiores a partir de um agente redutor mais fraco (p.ex. ácido ascórbico) e uma maior quantidade de íons $\mathrm{Ag}^{+}$adicionados ao meio (SAADE, 2013). Segundo Aherne (2008), a cinética da reação para a obtenção das AgNPs a partir das sementes pré-formadas não ocorre de forma reprodutível, podendo-se obter cristais com forma e tamanhos indefinidos, pois sementes com diferentes estruturas crescem em solução, e consequentemente, originam morfologias distintas ao final do procedimento. De maneira a se conseguir controlar a forma e o tamanho final de conjuntos de partículas coloidais é importante compreender o papel dos agentes surfactantes e sua influência no crescimento de AgNPs em meio aquoso. Por exemplo, a incorporação de surfactantes poliméricos no sistema, ainda na fase de formação da semente influencia na formação dos cristais no meio, estruturando-os quanto a sua forma e tamanho. Enquanto polímeros como o poliestireno sulfonato de sódio (PSSS) contribuem para a anisotropia do meio, gerando estruturas prismáticas como produto predominante, a polivinilpirrolidona (PVP), não iônico, estabilizam as AgNPs por um sistema de malha polimérica auto-organizada, impedindo a coalescência do sistema (MELO JR et al., 2012).

O presente estudo propõe a síntese de AgNPs utilizando concentrações variadas de sementes de (Ag-Ag)n associadas aos polímeros iônicos: PSSS, carbopol $940^{\circledR}$, quitosana, alginato de sódio e ao polímero não iônico álcool polivinílico (PVA) bem como o monitoramento de seu crescimento na presença destes polímeros.

\section{MATERIAIS E MÉTODOS}

Em um experimento típico, sementes de prata foram produzidas em meio aquoso combinando-se solução de citrato de sódio $\left(5 \mathrm{~mL}, 2,5 \mathrm{mmol} . \mathrm{L}^{-1}\right)$, solução do polímero desejado PSSS, quitosana peso molecular médio, PVA ou alginato de sódio, polifosfato de sódio, obtidos da Sigma Aldrich e carbopol $940^{\circledR}$ (C) obtido da Anastácio $\left(0,25 \mathrm{~mL}, 500 \mathrm{mg}\right.$. $\mathrm{L}^{-}$ $\left.{ }^{1}\right)$ e solução de borohidreto de sódio $\left(\mathrm{NaBH}_{4}\left(0,3 \mathrm{~mL}, 10 \mathrm{mmol} . \mathrm{L}^{-1}\right.\right.$, recém preparada), seguido pela adição de solução aquosa de $\mathrm{AgNO}_{3}\left(5 \mathrm{~mL}, 0,5 \mathrm{mmol} \cdot \mathrm{L}^{-1}\right.$, gota a gota). No crescimento combinou-se $5 \mathrm{~mL} \mathrm{H}_{2} \mathrm{O}$, ácido ascórbico $\left(75 \mu \mathrm{L}, 10 \mathrm{mmol}^{-\mathrm{L}^{-1}}\right)$ e volumes variados da semente $(40,60,90,120,200,260,400,500$ e $650 \mu \mathrm{L})$, seguido pela adição de solução aquosa de $\mathrm{AgNO}_{3}\left(3 \mathrm{~mL}, 0,5 \mathrm{mmol} . \mathrm{L}^{-1}\right)$. Os sistemas coloidais foram caracterizados por espectroscopia de absorção UV-Vis na região de 350 a $800 \mathrm{~nm}$.

\section{RESULTADOS E DISCUSSÃO}

Nos espectros de absorção das sementes, mostrados na Figura 1(a), com exceção dos coloides preparados na presença de quitosana e alginato, não foram observadas mudanças significativas. Nestas condições espera-se formação de nanopartículas em torno de 18-20 nm com absorção máxima em $\lambda=400 \mathrm{~nm}$ (SAADE, 2013). O resultado sugere que a quitosana não deve interagir forte o suficiente com os íons $\mathrm{Ag}^{+}$e os núcleos continuam a crescer resultando na banda de absorção larga, característica de uma grande dispersão de tamanho ou mesmo formando aglomerados. O alginato interferiu na 
formação das sementes, provavelmente prolongando o tempo de crescimento, resultando num tamanho maior d 30-40 nm (SAADE, 2013).

As Figuras 1(b) e (c) demonstram a tendência de crescimento das sementes no meio contendo os polímeros estudados, para o maior volume inicial de sementes $(650 \mu \mathrm{L})$ e o menor $(40 \mu \mathrm{L})$, respectivamente. Os resultados podem ser observados através de duas perspectivas: (i) quando incorporadas diferentes quantidades da semente ao sistema para um mesmo tipo de polímero e (ii) quando incorporadas quantidades equivalente de sementes para diferentes polímeros.

Figura 1 - Espectros de absorção das sementes de Ag (a) e das suspensões coloidais de AgNPs resultantes do crescimento com $650 \mu \mathrm{L}$ de semente (b) ou $40 \mu \mathrm{L}$ de semente (c) na presença dos diferentes polímeros.
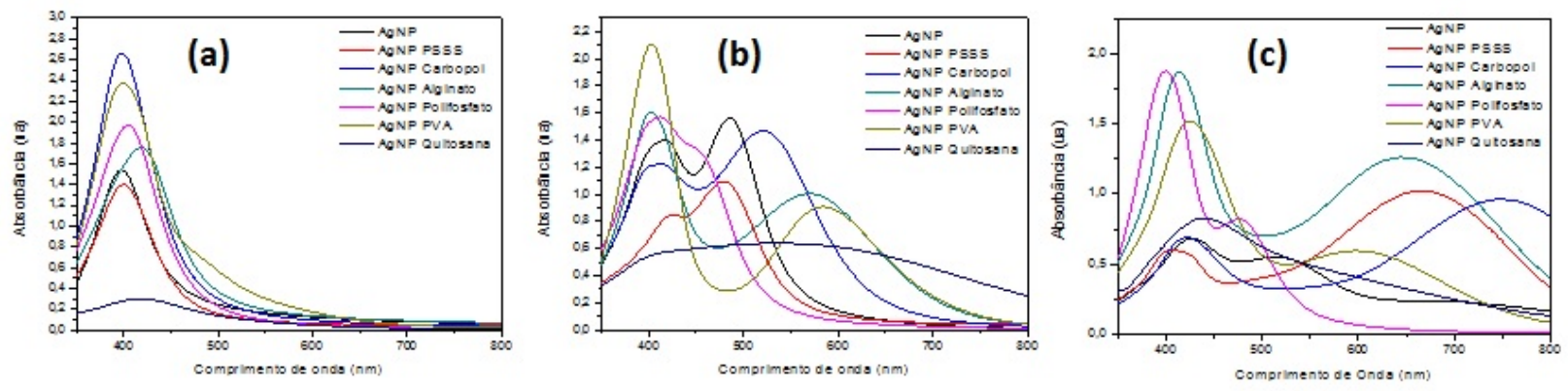

De uma forma geral, observam-se duas bandas após o crescimento das partículas: uma relacionada às sementes que restaram no meio (com máximos entre $400-430 \mathrm{~nm}$ ) e a outra referente às partículas que cresceram ou que aglomeraram (com máximos entre $450-750 \mathrm{~nm}$ ). Para todos os casos, uma maior concentração de sementes inicial resultou num menor deslocamento das bandas, ou seja, no crescimento mais rápido de um maior número de sementes, com o consumo rápido dos precursores adicionados. Em contrapartida, observa-se que a partir de uma menor concentração inicial de sementes ocorreu um maior deslocamento das bandas para o vermelho, indicando um crescimento lento, com a adição de íons $\mathrm{Ag}^{+}$às sementes iniciais e o crescimento gradual destas. Isso se observa independente do polímero utilizado, à exceção da quitosana que levou à turvação do sistema, indicando aglomeração, para todas as condições. As partículas de Ag que não possuíam agente surfactante formaram agregados para o volume de semente menor inicial, mas mantiveram-se estáveis com o volume inicial maior de sementes, provavelmente devido à estabilização decorrente da adsorção de ácido cítrico em sua superfície.

Não houve correlação entre a existência de carga ou não no polímero utilizado e o padrão de crescimento das partículas. No meio contendo o PVA, os resultados mostraram uma maior estabilização das sementes de prata, ocorrendo mínimo consumo desse sistema para o subsequente crescimento das AgNPs. O Polifosfato, altamente ionizado não resultou num crescimento de partículas pronunciado, para nenhuma das quantidades iniciais adicionadas, além de proporcionar um perfil bimodal de tamanho de partículas. Em contraste, as partículas submetidas à presença de carbopol, PSSS e alginato cresceram de forma extensiva e mantiveram um perfil espectral semelhante, sugerindo uma morfologia também semelhante, neste caso, o de discos prismáticos (AHERNE et al., 2008). Este resultado, observado para o PSSS foi demonstrado por Aherne e colaboradores (2008) como sendo decorrente da associação deste polímero preferencialmente a uma das faces cristalinas das sementes de Ag, induzindo a anisotropia do meio durante o crescimento posterior. 


\section{CONCLUSÃO}

Conclui-se que as AgNPs na presença de diferentes polímeros surfactantes, demonstram diferentes modelos de crescimento, que podem ser associados às interações moleculares/carga com a superfície das sementes. As AgNPs crescidas na presença dos polímeros carbopol e alginato mostraram cinética reacional semelhantes à observada para o PSSS, sugerindo o uso destes agentes poliméricos biocompatíveis para a estabilização de nanopartículas de Ag para futuras aplicações, principalmente, biomédicas e fotônicas.

\section{REFERÊNCIAS}

AHERNE, D.; LEDWITH, D. M.; GARA, M.; KELLY, J. M. Optical Properties and Growth Aspects of Silver Nanoprisms Produced by a Highly Reproducible and Rapid Synthesis at Room

Temperature. Advanced Functional Materials. v. 18, p 2005-2016, 2008.

MELO JR et al. Preparação de nanopartículas de prata e ouro: um método simples para a introdução da nanociência em laboratório de ensino. Química Nova. v.35, no.9, 2012.

SAADE, J. Síntese/Fabricação e caracterização de micro e nanoestruturas para aplicação na fotônica e plasmônica. 134f. Dissertação (Doutorado), Centro De Ciências Exatas e da Natureza, Universidade Federal de Pernambuco, Recife, 2013

\section{AGRADECIMENTOS}

Os autores são gratos ao $\mathrm{CNPq}$ pela bolsa de estudo, e às centrais analíticas do Departamento de Química Fundamental (DQF) e Departamento de Ciências Farmacêuticas (DCFar) da UFPE pela permissão para utilização de equipamentos. Este trabalho faz parte de um projeto financiado pelo CNPq através do Instituto de Ciência e Tecnologia em Fotônica (INCT-INFo). 